\title{
iDrug: a web-accessible and interactive drug discovery and design platform
}

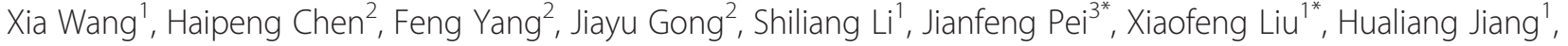 \\ Luhua Lai ${ }^{3}$ and Honglin Li, ${ }^{1,2^{*}}$
}

\begin{abstract}
Background: The progress in computer-aided drug design (CADD) approaches over the past decades accelerated the early-stage pharmaceutical research. Many powerful standalone tools for CADD have been developed in academia. As programs are developed by various research groups, a consistent user-friendly online graphical working environment, combining computational techniques such as pharmacophore mapping, similarity calculation, scoring, and target identification is needed.

Results: We presented a versatile, user-friendly, and efficient online tool for computer-aided drug design based on pharmacophore and 3D molecular similarity searching. The web interface enables binding sites detection, virtual screening hits identification, and drug targets prediction in an interactive manner through a seamless interface to all adapted packages (e.g., Cavity, PocketV.2, PharmMapper, SHAFTS). Several commercially available compound databases for hit identification and a well-annotated pharmacophore database for drug targets prediction were integrated in iDrug as well. The web interface provides tools for real-time molecular building/editing, converting, displaying, and analyzing. All the customized configurations of the functional modules can be accessed through featured session files provided, which can be saved to the local disk and uploaded to resume or update the history work.
\end{abstract}

Conclusions: iDrug is easy to use, and provides a novel, fast and reliable tool for conducting drug design experiments. By using iDrug, various molecular design processing tasks can be submitted and visualized simply in one browser without installing locally any standalone modeling softwares. iDrug is accessible free of charge at http://lilab.ecust.edu.cn/idrug.

Keywords: Online drug design platform, Cavity detection, Pharmacophore search, 3D similarity calculation, Target prediction

\section{Background}

Computer-aided drug design (CADD) is a widely used term that represents computational tools and sources for the storage, management, analysis and modeling of compounds [1]. Benefiting from the dramatic increase of biomacromolecular and small molecular information, CADD techniques are used at various stages of a drug-discovery project, from target identification and validation to lead discovery and optimization, even extending to preclinical trials [2].

\footnotetext{
*Correspondence: jfpei@pku.edu.cn; xfliu@ecust.edu.cn; hlli@ecust.edu.cn ${ }^{3}$ BNLMS, Center for Quantitative Biology, State Key Laboratory for Structural Chemistry of Unstable and Stable Species, College of Chemistry and Molecular Engineering, Peking University, Beijing 100871, China

'Shanghai Key Laboratory of New Drug Design, State Key Laboratory of Bioreactor Engineering, School of Pharmacy, East China University of Science and Technology, Shanghai 200237, China

Full list of author information is available at the end of the article
}

A huge body of CADD software has been developed over the years in many different research groups [3-11]. However, most molecular design packages, with single client interface and powerful algorithms, may require separate license keys to be purchased individually. In addition, due to the complexity and diversity of molecular design procedures, sometimes even trained computational chemists can not perfectly excel in what they are computing [12]. In parallel, the open source and easily accessible software, which provides a great opportunity to perform research in CADD, has often encountered two main problems when being used by medicinal chemists [13]. First, in many instances the authors of CADD software pay more attention to scientific details rather than the usability of software, thus making it rarely associated with the graphical user interface and difficult to use for non-expert experimentalists. Second, individual
() Chemistry Central 
program usually requires a specific input structure and produces a specific output format, making users incapable of combining different programs.

The striking growth of web technologies provides an alternative strategy to offer the possibility of accessing and running computational chemistry tools directly on the web with a simplified user interface. The major advantages of these web-based solutions are that users can submit models and data to the online servers without regularly downloading and updating the data collections and tools to their local disks. An increasing number of web applications for performing molecular modeling and processing are also available for end users [14-17]. However, these tools are always not able to integrate various molecular design programs and enormous data collections to meet the systematic operations of users to achieve the best results [18]. Besides, even if some initiatives combining different computational programs exists, e.g. for e-LEA3D [19], Sanjeevini [20], only focused on structure-based drug design such as molecular docking and de novo drug design which rely on the knowledge of the given structure of the target macromolecule.

Here, we developed an online interactive platform, termed $i$ Drug, to break expensive commercial suites and command line barrier and introduce a user-friendly web environment to conduct 3D molecular similarity calculation and to construct pharmacophore models for virtual screening. The user may select the molecular processing engine used by $i$ Drug and setup and initiate jobs. Currently the system allows access to derive pharmacophore models directly from the given receptor regardless of a molecular $3 \mathrm{D}$ structure in the corresponding binding pose conformation provided as the reference (Cavity [8] and PocketV2 [21]); generate novel molecules for the given site using pharmacophore mapping approach; predict targets of a chemical of interest such as drugs, lead compounds and natural products (PharmMapper [22]); and rank candidates based on similarity-based database searching (SHAFTS $[7,23,24])$. Different modules described above (Table 1 ) have

Table 1 List of computational techniques supported by iDrug

\begin{tabular}{llll}
\hline Name & Method & Refs & $\begin{array}{l}\text { Free for } \\
\text { academia }\end{array}$ \\
\hline Cavtiy & $\begin{array}{l}\text { Detect and score potential binding } \\
\text { sites of a protein } \\
\text { Derive pharmacophore models } \\
\text { bocket v.2 }\end{array}$ & $\begin{array}{l}{[8,33]} \\
\text { based on a given receptor of } \\
\text { complex structure }\end{array}$ & Yes \\
PharmMapper & $\begin{array}{l}\text { Pharmacophore mapping } \\
\text { (online web service) }\end{array}$ & {$[22]$} & Yes \\
SHAFTS & 3D similarity calculation & {$[7,23]$} & Yes \\
Cyndi & Molecular conformation generation & {$[38,39]$} & Yes \\
Pybel & $\begin{array}{l}\text { Python wrapper for the OpenBabel } \\
\text { cheminformatics toolkit }\end{array}$ & {$[25,26]$} & Yes \\
\hline
\end{tabular}

been incorporated, which work in a pipeline as depicted in the architecture (Figure 1). It also features a session based working bench to save, resume, and reuse the jobs and configurations customized by users, which can be accessed and updated through the interface easily. As $i$ Drug addresses common problems associated with either biomacromolecule or small molecule, it is expected to help both experts and non-specialists to achieve the automated molecular design of daily research demands, as well as being a routine adjunct to experimental studies.

\section{Methods}

\section{Implementation and interface}

The $i$ Drug system was developed using Java on Apache Tomcat Server. The platform back-end was developed in Python programming language, with MySQL as the database system, while the front-end is implemented with JSP using JavaScript and AJAX technologies utilizing the jQuery library. The open-source Java viewer Jmol (http://www.jmol.org/) is used for 3D display and manipulation of molecular structures. Pybel [25,26], a Python wrapper of the OpenBabel toolkit, was used in backend for molecular file parsing and converting.

The user interface of $i$ Drug is shown in Figure 2. Users can normally start a session by clicking the 'Load' button. As $i$ Drug provides pharmacophore- and similarity-based tasks, the corresponding uploading dialog is grouped into two modes: 'pharmacophore work mode' and 'similarity work mode'. After a successful submission, a unique Job ID is assigned and used to access the computational results. Result files associated with the completed jobs are stored at the server for 3 months, which can be downloaded for offline analysis within this period of time.

\section{Chemical and drug target database}

$i$ Drug currently integrates the publicly accessible $\mathrm{NCI}$ database [27] and the commercially available and biological relevant compounds from ZINC [28], comprising over 3 million compounds with multiple 3D conformers pregenerated to facilitate both pharmacophore and 3D similarity based virtual screening. An in-house pharmacophore database called PharmTargetDB [22] containing over 7,000 pharmacophore models derived from complex crystal structures with corresponding protein target annotations is incorporated in $i$ Drug as well. The target protein annotations including name, function, and involved indication/ disease were referred from DrugBank [29], BindingDB [30], PDBBind [31], and PDTD [32] databases. The key information for each database was summarized in Table 2.

\section{Cavity detection and structure-based pharmacophore modeling}

Identification of protein cavities is of fundamental importance for structure-based pharmacophore derivation. 


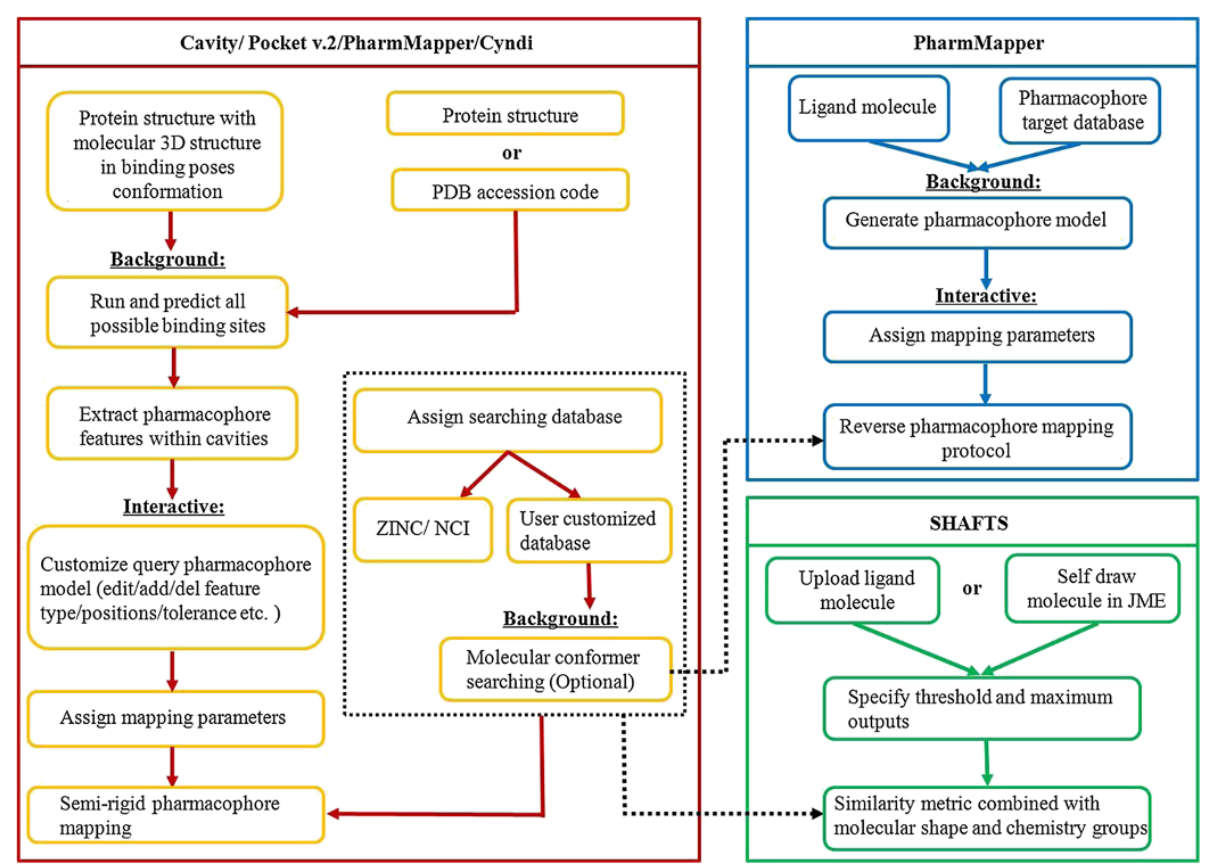

Figure 1 Workflow of iDrug automating pharmacophore modeling using Cavity and Pocket v.2, screening with PharmMapper and SHAFTS, and searching conformers using Cyndi. Common modules in iDrug platform are framed in black.

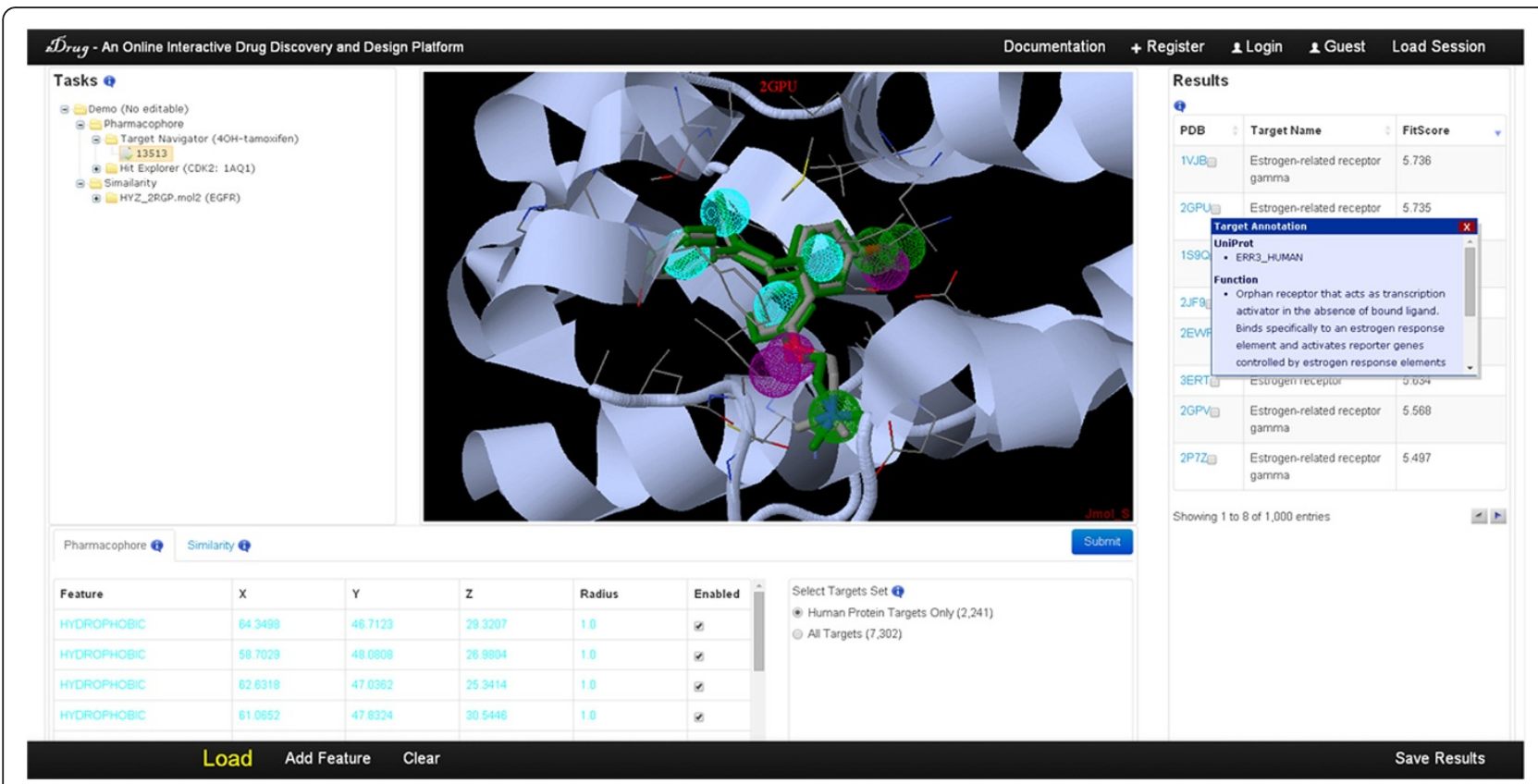

Figure 2 The iDrug interface. The task management is in the upper left and provides easy access to the full set of the history work. The Jmol-based molecular viewer is in the middle and displays the query molecule and results structure. The query editor is shown in the bottom and supports the interactive modification of the parameters based on the properties of the computational software. The results browser is on the right and displays the complete results along with the available details. In this figure, potential targets of tamoxifen obtained from iDrug are shown as well as the target pharmacophore model. On mouse over, a preview of the annotation information is displayed in a pop-up window, as shown for 2GPU in this example. 
Table 2 Data source of $i$ Drug

\begin{tabular}{ccc}
\hline Type & Database & Number of entities \\
\hline Compounds & ZINC Lead-like & $3,027,615$ \\
& NCl Open Database & 246,483 \\
Targets & PharmTargetDB & 7,302 \\
\hline
\end{tabular}

$i$ Drug applies Cavity to detect the potential binding sites on the surface of a given protein structure, and rank them according to the corresponding druggability scores on the basis of geometric shape, hydrogen bonding, and hydrophobic effects. By comparison of the success rate for the first-ranked prediction tested on the PDBBind dataset, Cavity outperformed four popularly used binding sites detection approaches (LIGSITEcsc, Q-sitefinder, SURFNET, and PASS), indicating its accurate prediction of relatively small binding sites on the surface of a protein [8,33]. Li et al. customized LigBuilder 2.0 to generate and fit the shape of the Cyclophilin A (CypA) binding site and finally obtained two highly potent inhibitors with nanomolar inhibitory potencies (2.59 $\mathrm{nM}$ and $1.52 \mathrm{nM}$ ) [34]. A receptor-based pharmacophore modeling program Pocket v.2 was used to extract pharmacophore features within cavities [21]. In this approach, hotspot analysis and grid-based scoring were used to identify essential pharmacophore features. As suggested by case study results, Pocket v.2 can yield consistent pharmacophore models for proteins with minor conformational changes upon binding of different ligands, indicating the robustness of the approach. Lai et al. proposed a novel strategy that combined receptor-based common pharmacophores with molecular docking. Three compounds were identified through this strategy to inhibit LTA4H-h and hnps-PLA2 simultaneously [35].

Normally, $i$ Drug requires a protein $3 \mathrm{D}$ structure or a known PDB accession code as input to derive pharmacophore models. All binding sites along with the extracted pharmacophore features will be displayed and ranked by the druggability scores. A small molecular $3 \mathrm{D}$ structure in the corresponding binding poses conformation can also be uploaded as the reference to specify the position of the binding site and generate the pharmacophore features representing the corresponding interaction mode. It takes averagely 1-3 minutes for binding sites detection, and the potential binding sites can be interactively viewed and downloaded in PDB files for off-line analysis, which includes the binding pocket surface, the residues around the site, and key features of the pharmacophore model derived from the site.

\section{Pharmacophore mapping and virtual screening}

For pharmacophore-based screening, $i$ Drug employs a pharmacophore mapping method called PharmMapper based on feature triplet hashing and searching algorithm [22]. Liu et al. discovered two potent IGF-1R kinase inhibitors via hierarchical strategy based virtual screening (pharmacophore screening and docking), which efficiently reduced the number of "nonhits" passed to docking stage and consequently reduced the computational cost [36].

The query pharmacophore model can be derived from the potential binding sites detected previously or user customized ones. $i$ Drug allows interactive editing of the features of the pharmacophore query like feature type, positions, and size of the tolerance spheres. Pharmacophore searching is performed against the available or user uploaded databases. The hit compounds are compiled into the table with the corresponding original weblinks whereas applicable, and ranked by the fit values with the query. The superimposed mode of the molecules onto the pharmacophore query along with the corresponding binding site can be interactively visualized in Jmol applet.

\section{Target identification}

$i$ Drug uses the reverse pharmacophore mapping procedure to predict potential drug targets. Qian et al. discovered a novel series of acenaphtho[1,2-b]pyrrole derivatives as potent FGFR1 inhibitors by using this in silico targets screening approach [37]. The results were subsequently validated by enzyme-linked immunosorbent assay. The platform takes a single drug-like molecule or natural product chemical structure as an input and automatically perceives all chemotype features of the query molecule. Users are allowed to modify the perceived pharmacophore model and search the pharmacophore target database with it. The results can also be filtered in terms of both the number of returned results and the properties of the algorithm. Since the geometric matching alignment and scoring method uses rigid conformations, multiple conformers of each query molecule must be generated. In-house program Cyndi [38,39], which uses a multi-objective evolution algorithm method for conformer searching, was chosen by default to generate multiple conformation. Additionally, the minimum number of each pharmacophore feature type and a fit score cutoff can be specified to discard those target pharmacophore models, of which the corresponding values are less than the threshold. On average, the total time consumed by the complete screening and scoring protocol ranges from 1-2 $\mathrm{h}$ depending on the flexibility of the input molecule and filter parameters assigned by the user.

Upon completion of the computations, the results of the hit target pharmacophore models are demonstrated in the form of a ranked list. Each row contains the protein ID, which is a hyperlink points to the PDB website [40], the target name, the fit values between the small molecule and the pharmacophores, and a unique orientation of a conformation of the query along with 
the $3 \mathrm{D}$ structure information of the target visualized by the Jmol applet. Detailed annotations of the hit targets are presented in a pop-up window when the cursor moves over the corresponding PDB codes. Alternatively, a downloadable zip file containing the aligned pose with the corresponding pharmacophore model and the complete targets information in CSV format is accessible for each returned match.

\section{Similarity search}

For the implementation of ligand-based searching, $i$ Drug adopts an efficient 3D similarity calculation method SHAFTS, which is designed to integrate the strength of pharmacophore matching and volumetric overlay approaches [7]. Hits are determined by a hybrid similarity score cut-off and alignment poses of compounds will be generated by enumerating all potential pharmacophore feature triplets matches. Liu et al. discovered sixteen compounds with $\mathrm{IC}_{50}<20 \mu \mathrm{M}$, three of which showed low micromolar inhibitory activities against p90 ribosomal S6 protein kinase 2 (RSK2) and exhibited selectivity across a panel of related kinases using SHAFTS [23]. By adopting the same strategy, $\mathrm{Xu}$ et al. reported a novel pteridin-7(8H)-one epidermal growth factor receptor (EGFR) inhibitor scaffold with potent and selective inhibitory activity against both wild-type and T790M/ L858R mutant EGFRs. The most remarkable agent showed highly inhibitory activity against the growth of gefitinib-resistant H1975 cells, making it a potential lead for further development of EGFR kinase-related anticancer drugs [41]. The results suggests that SHAFTS is an efficient and powerful tool in scaffold hopping and hit identification endeavors. Moreover, Shen et al. used the evodiamine derivative as a probe to search MDL/Symyx Drug Data Report (MDDR) [42] with ChemMapper. The fourth ranked protein, topoisomerase II (Top2), was a well-known antitumor target. Relaxation assay proved that the analogs of the natural product are potent inhibitor against Top2, with stronger activity than the well-known Top $2 \alpha$ inhibitor etoposide [43].

A chemical structure (sketched online or uploaded in multiple chemical structure file formats) is accepted as the query to perform similarity searching. It takes hours to days per search depending on the complexity of the query molecule and the size of the library.

The outputs are generated as a list of compounds sorted by similarity scores to the query and can be downloaded in a mol2 file. The superimposed pose of each hit with the query can be visualized interactively in Jmol applet along with the molecular surface representation and perceived pharmacophore features by SHAFTS.

\section{Results and discussion}

\section{Benchmarking study}

To demonstrate potential applications of $i$ Drug platform, we performed pharmacophore-based virtual screening libraries with the MUV data sets [44], molecular 3D similarity-based virtual screening with the enhanced Directory of Useful Decoys (DUD-E) [45] data sets, and reversed pharmacophore mapping-based drug target identification with the pharmacophore target database. Receiver Operator Characteristic (ROC) curves, Area Under the ROC Curves (AUC), and enrichment factors (EF) were calculated after ranking compounds from the MUV and DUD-E data sets. EF after $\mathrm{x} \%$ of the library screened were calculated according to the following formula (Hits $_{\text {sampled }}=$ number of hits found at $\mathrm{x} \%$ of the database screened, $N_{\text {sampled }}=$ number of compounds screened of $\mathrm{x} \%$ of the database, $N_{\text {total }}=$ the number of compounds in the entire database, Hits $_{\text {total }}=$ the number of actives in the entire database).

$$
\mathrm{EF}=\frac{\text { Hits }_{\text {Sampled }}}{N_{\text {Sampled }}} \times \frac{N_{\text {Total }}}{\text { Hits }_{\text {Total }}}
$$

\section{Case 1: pharmacophore-based virtual screening}

CDK2 (Cyclin-dependent kinase 2) is a protein kinase whose pharmacophore features, depicting ligands that target against the ATP binding site, are well described in the literature [46]. We used $i$ Drug to create pharmacophore queries from the crystal structures of CDK2 (PDB:1AQ1). The pharmacophore queries occupied by the bioactive conformation of the ligand, which contain one $\mathrm{H}$-bond

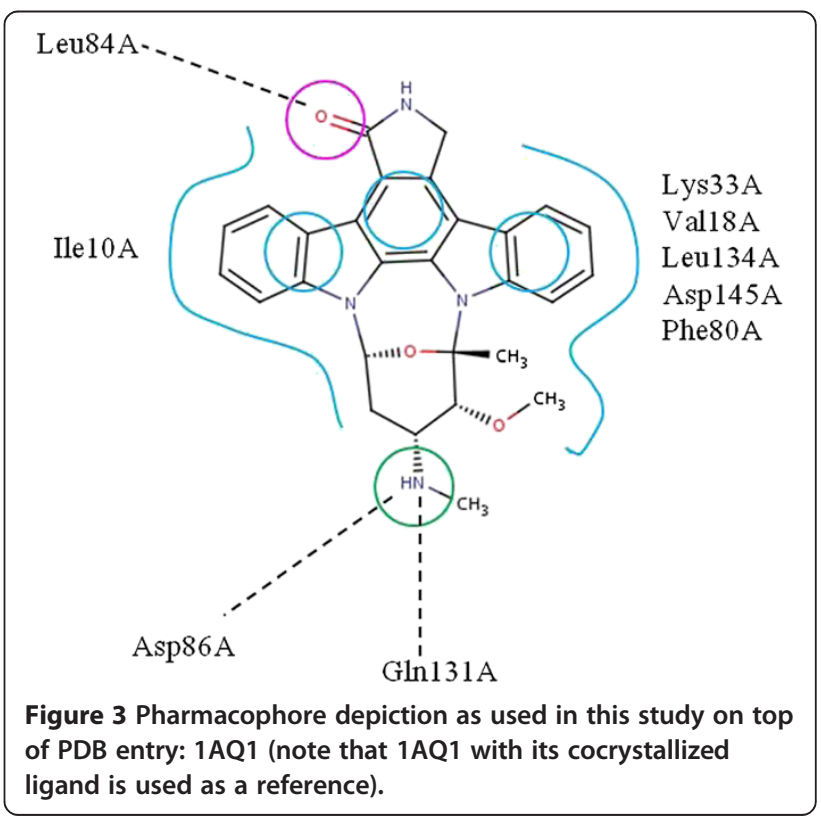


Table 3 AUC value and EF values at 0.5, 1, 2 and 5\% for CDK2 inhibitor pharmacophore-based virtual screening

\begin{tabular}{lcccc}
\hline AUC & \multicolumn{5}{c}{ EF } \\
\hline 0.63 & $0.5 \%$ & $1.0 \%$ & $2.0 \%$ & $5.0 \%$ \\
& 2.5 & 1.3 & 2.5 & 2 \\
\hline
\end{tabular}

acceptor, one $\mathrm{H}$-bond donor and three hydrophobic features, were selected as the hypotheses (Figure 3).

We create the compound sets using the virtual screening dataset of CDK2 comprising 80 active compounds and 15000 decoy compounds [47]. The search of 249,242 conformers of 15080 compounds takes almost 20 minutes. Without any prescreening, $i$ Drug matches 50 out of 80 actives and 5641 out of 15000 decoys resulting in an enrichment factor of 1.7. The AUC value is 0.63 , indicating that the overall enrichment is only slightly better than that expected from a random selection (Table 3). This is an important observation suggesting that though not so effective in actives enrichment with single pharmacophore model, $i$ Drug enables fast prefiltering for large compound collections before applying more accurate and computationally expensive algorithms.

\section{Case 2: target prediction}

Tamoxifen, which is used as an adjuvant therapy in the treatment of breast cancer, has been proved as a multiple target drug. So far, tamoxifen and its active metabolite, 4OH-tamoxifen, are known to interact with 16 targets. We have chosen to investigate $4 \mathrm{OH}$-tamoxifen as a test case for validation (the typical run takes less than an hour). The 11 known targets of $4 \mathrm{OH}$-tamoxifen retrieved by $i$ Drug are shown in Table 4 . Other targets, including microsomal antiestrogen binding site (AEBS) [48],

\section{Table 4 Retrieval of 11 targets of $40 \mathrm{H}-T a m o x i f e n$ by} iDrug

\begin{tabular}{|c|c|c|c|c|}
\hline Target name & Reference & Score & $\begin{array}{l}\text { PDB } \\
\text { ID }\end{array}$ & $\begin{array}{c}\text { Rank } \\
(\%)\end{array}$ \\
\hline Estrogen receptor- $\gamma$ & {$[52]$} & 5.736 & $1 \mathrm{VJB}$ & 0.01 \\
\hline $\begin{array}{l}\text { Estradiol } 17 \beta \text {-hydroxysteroid } \\
\text { dehydrogenase } 1\end{array}$ & [53] & 4.111 & $115 R$ & 0.26 \\
\hline Dihydrofolate reductase & [54] & 3.777 & 1DG7 & 0.45 \\
\hline Glutathione S-transferase A1 & {$[55]$} & 3.655 & $1 \mathrm{GSF}$ & 0.73 \\
\hline Prostaglandin G/H synthase 2 & {$[56]$} & 3.411 & $1 P X X$ & 1.36 \\
\hline Liver carboxylesterase 1 & [57] & 3.344 & 1YA4 & 1.59 \\
\hline Protein kinase $C$ theta type & {$[58]$} & 3.171 & $1 X J D$ & 2.18 \\
\hline Calmodulin & [59] & 2.974 & 1XA5 & 3.63 \\
\hline Collagenase 3 & {$[60]$} & 2.945 & $317 \mid$ & 4.57 \\
\hline Alcohol dehydrogenase E chain & {$[60]$} & 2.881 & $1 \mathrm{MGO}$ & 7.37 \\
\hline $\begin{array}{l}\text { 3-alpha-(or20-beta)-hydroxysteroid } \\
\text { dehydrogenase }\end{array}$ & [61] & 2.835 & $1 \mathrm{HDC}$ & 9.87 \\
\hline
\end{tabular}

cholesterol acyl transferase (ACAT) [48], cholesterol epoxide hydrolase [49], hedgehog signaling [50], immunoglobulin [51], are missed due to the limited coverage of the pharmacophore database. In spite of this, the reverse pharmacophore mapping approach, therefore, enables 11 of 16 experimentally confirmed tamoxifen targets to be retrieved within $10 \%$ of the ranked database, which is promising and reliable for a retrospective target identification case.

\section{Case 3: molecular 3D similarity-based screening}

The crystal ligand structure of epidermal growth factor receptor (EGFR, PDB:2RGP) was searched via $i$ Drug. The screening library was created using the DUD-E data sets including 542 active compounds and 35,050 decoy compounds for a baseline enrichment of $1.5 \%$. A multiconformer library was generated using Cyndi with the default settings (up to 200 conformers per a compound), resulting in a library of 2,735,015 conformations.

The search of EGFR query in $i$ Drug takes 12-14 hours. The performance of $i$ Drug with respect to compound library enrichment, i.e., the fraction of true positives versus the fraction of false positives, was visualized in ROC. The corresponding AUC and enrichment values at $0.5,1.0,2.0$, and $5.0 \%$ are calculated and reported, which can be found in Table 5. The AUC value is 0.87 and the $\mathrm{EF}$ value at the $0.5 \%$ level is 56.9 , indicating highly significant enrichment with respect to random ones.

\section{Experimental}

\section{Compound data sets}

Datasets from four different public sources were used. A set of over 3 million compounds extracted from ZINC [28] and NCI [27] were used for both pharmacophore and 3D similarity based virtual screening. Cyndi [38] was used to generate a maximum of 50 low-energy conformations for each compound. The MUV data sets are based on bioactivity data. MUV consists of 17 targets, each with 30 actives and 15000 decopys [44]. In this study we reproduced the evaluation of Sanders et al [47] and derived pharmacophore queries from the crystal structure of CDK2 (PDB:1AQ1). The DUD-E was originally designed for benchmarking of docking methods [45]. A subset was later extracted for the use in 3D similaritybased virutal screening experiments. The DUD-E contains 102 targets with 22886 clustered ligands drawn from ChEMBL, each with 50 property-matched decoys drawn

Table 5 AUC value and EF values at $0.5,1,2$ and $5 \%$ for EGFR inhibitor similarity virtual screening

\begin{tabular}{lllll}
\hline AUC & \multicolumn{5}{c}{ EF } \\
\hline 0.87 & $0.5 \%$ & $1.0 \%$ & $2.0 \%$ & $5.0 \%$ \\
& 56.9 & 42.9 & 28.5 & 13.6 \\
\hline
\end{tabular}


from ZINC. In this study EGFR with 542 actives were used. Besides, for the user uploaded target compounds, multi conformations (at most 100) would be generated online with Cyndi prior to virtual screening if the 'Generate Conformers' is toggled on.

\section{Target pharmacophore databases}

The target protein structures co-complexed with small molecules were selected from DrugBank, BindingDB, PDBBind, and PDTD databases. All the small ligands with molecular weight lower than 100 , such as solvents, buffers and metal cations, and all the cofactors with molecular weight over 600, such as CoAs, polypeptides and nucleic acids were regarded. For the proteins existing as homopolymers, only one monomer was reserved for analysis. For the proteins determined by NMR with multiple structure models, only the first model was selected for pharmacophore generation. LigandScout [9] was used in the process of pharmacophore model derivation. As a result, a database with 7302 pharmacophore models were generated and stored in $i$ Drug. This database has been used in our previous publications [22].

\section{Conclusions}

We presented $i$ Drug, a versatile web server for both pharmacophore- and similarity-based virtual screening and target identification to facilitate computational drug discovery. The interface is easy-to-use and can be accessed by user customized sessions to free them from installing standalone softwares. $i$ Drug provides ready-to-access compounds and pharmacophore target databases for virtual screening and target identification. Various applications like binding site identification, structure-based pharmacophore derivation, conformational sampling, pharmacophore searching, and 3D similarity calculation are integrated as individual modules. It enables interactive editing and refinement of pharmacophore hypothesis as well as flexible customization of the parameters. The featured job management system ensures the user privacy and project tracking through a session-based mechanism.

\section{Competing interests}

The authors declare that they have no competing interests.

\section{Authors' contributions \\ $\mathrm{HL}$ designed the project. XW analyzed the data, contributed the use cases and wrote the manuscript. HC and FY supported the project with background and front-end development. JG contributed the back-end schedule system. JP, $X \mathrm{~L}, \mathrm{HJ}, \mathrm{LL}$, and $\mathrm{HL}$ contributed the molecular design approaches. All authors read and approved the final manuscript.}

\section{Acknowledgments}

This work was supported by the $863 \mathrm{Hi}$-Tech Program of China (grant 2012AA020308), the National Natural Science Foundation of China (grants 81230090, 81222046 and 81230076), the Shanghai Committee of Science and Technology (grant 12401900801), and the National S\&T Major Project of China (Grants 2013ZX09507004). Honglin Li is also sponsored by
Shanghai Rising-Star Tracking Program (grant 13QH1401100) and Fok Ying Tung Education Foundation (141035).

\section{Author details}

'Shanghai Key Laboratory of New Drug Design, State Key Laboratory of Bioreactor Engineering, School of Pharmacy, East China University of Science and Technology, Shanghai 200237, China. ${ }^{2}$ School of Information Science and Engineering, East China University of Science and Technology, Shanghai 200237, China. ${ }^{3}$ BNLMS, Center for Quantitative Biology, State Key Laboratory for Structural Chemistry of Unstable and Stable Species, College of Chemistry and Molecular Engineering, Peking University, Beijing 100871, China.

Received: 20 March 2014 Accepted: 12 May 2014

Published: 23 May 2014

\section{References}

1. Song CM, Lim SJ, Tong JC: Recent advances in computer-aided drug design. Brief Bioinform 2009, 10:579-591.

2. Jorgensen WL: The many roles of computation in drug discovery. Science 2004, 303:1813-1818.

3. Kellenberger E, Foata N, Rognan D: Ranking targets in structure-based virtual screening of three-dimensional protein libraries: methods and problems. J Chem Inf Model 2008, 48:1014-1025.

4. Li YY, An J, Jones SJ: A computational approach to finding novel targets for existing drugs. PLoS Comput Biol 2011, 7:e1002139.

5. Nettles JH, Jenkins JL, Bender A, Deng Z, Davies JW, Glick M: Bridging chemical and biological space: "target fishing" using 2D and 3D molecular descriptors. J Med Chem 2006, 49:6802-6810.

6. Campillos M, Kuhn M, Gavin A-C, Jensen L, Bork P: Drug target identification using side-effect similarity. Science 2008, 321:263-266.

7. Liu X, Jiang H, Li H: SHAFTS: a hybrid approach for 3D molecular similarity calculation. 1. Method and assessment of virtual screening. J Chem Inf Model 2011, 51:2372-2385.

8. Yuan Y, Pei J, Lai L: LigBuilder 2: a practical de novo drug design approach. J Chem Inf Model 2011, 51:1083-1091.

9. Wolber G, Langer T: LigandScout: 3-D pharmacophores derived from protein-bound ligands and their use as virtual screening filters. J Chem Inf Model 2005, 45:160-169.

10. Vainio MJ, Puranen JS, Johnson MS: ShaEP: molecular overlay based on shape and electrostatic potential. J Chem Inf Model 2009, 49:492-502.

11. Kurogi Y, Guner OF: Pharmacophore modeling and three-dimensional database searching for drug design using catalyst. Curr Med Chem 2001, 8:1035-1055.

12. Stouch TR: The errors of our ways: taking account of error in computer-aided drug design to build confidence intervals for our next 25 years. J Comput Aided Mol Des 2012, 26:125-134.

13. Lill MA, Danielson ML: Computer-aided drug design platform using PyMOL. J Comput Aided Mol Des 2011, 25:13-19.

14. Li H, Gao Z, Kang L, Zhang H, Yang K, Yu K, Luo X, Zhu W, Chen K, Shen J, Wang $X$, Jiang H: TarFisDock: a web server for identifying drug targets with docking approach. Nucleic Acids Res 2006, 34:W219-W224.

15. Gong J, Cai C, Liu X, Ku X, Jiang H, Gao D, Li H: ChemMapper: a versatile web server for exploring pharmacology and chemical structure association based on molecular 3D similarity method. Bioinformatics 2013, 29:1827-1829.

16. Koes DR, Camacho CJ: ZINCPharmer: pharmacophore search of the ZINC database. Nucleic Acids Res 2012, 40:W409-W414.

17. Schmidtke P, Le Guilloux V, Maupetit J, Tuffery P: fpocket: online tools for protein ensemble pocket detection and tracking. Nucleic Acids Res 2010, 38:W582-W589.

18. Rarey M: Some thoughts on the " $\mathrm{A}$ " in computer-aided molecular design. J Comput Aided Mol Des 2012, 26:113-114.

19. Douguet D: e-LEA3D: a computational-aided drug design web server. Nucleic Acids Res 2010, 38:W615-W621.

20. Jayaram B, Singh T, Mukherjee G, Mathur A, Shekhar S, Shekhar V: Sanjeevini: a freely accessible web-server for target directed lead molecule discovery. BMC Bioinforma 2012, 13(Suppl 17):S7.

21. Chen J, Lai L: Pocket v. 2: further developments on receptor-based pharmacophore modeling. J Chem Inf Model 2006, 46:2684-2691.

22. Liu X, Ouyang S, Yu B, Liu Y, Huang K, Gong J, Zheng S, Li Z, Li H, Jiang H: PharmMapper server: a web server for potential drug target 
identification using pharmacophore mapping approach. Nucleic Acids Res 2010, 38:W609-W614.

23. Lu W, Liu X, Cao X, Xue M, Liu K, Zhao Z, Shen X, Jiang H, Xu Y, Huang J, Li H: SHAFTS: a hybrid approach for 3D molecular similarity calculation. 2 . Prospective case study in the discovery of diverse p90 ribosomal 56 protein kinase 2 inhibitors to suppress cell migration. J Med Chem 2011, 54:3564-3574.

24. Bai F, Liu H, Tong L, Zhou W, Liu L, Zhao Z, Liu X, Jiang H, Wang X, Xie H: Discovery of novel selective inhibitors for EGFR-T790M/L858R. Bioorg Med Chem Lett 2012, 22:1365-1370.

25. O'Boyle NM, Morley C, Hutchison GR: Pybel: a Python wrapper for the OpenBabel cheminformatics toolkit. Chem Cent J 2008, 2:5.

26. O'Boyle NM, Banck M, James CA, Morley C, Vandermeersch T, Hutchison GR: Open Babel: an open chemical toolbox. J Cheminform 2011, 3:1-14.

27. Ihlenfeldt W-D, Voigt JH, Bienfait B, Oellien F, Nicklaus MC: Enhanced CACTVS browser of the Open NCI Database. J Chem Inf Comput Sci 2002, 42:46-57.

28. Irwin JJ, Shoichet BK: ZINC-a free database of commercially available compounds for virtual screening. J Chem Inf Model 2005, 45:177-182.

29. Wishart DS, Knox C, Guo AC, Cheng D, Shrivastava S, Tzur D, Gautam B, Hassanali M: DrugBank: a knowledgebase for drugs, drug actions and drug targets. Nucleic Acids Res 2008, 36:D901-D906.

30. Liu T, Lin Y, Wen $X$, Jorissen RN, Gilson MK: BindingDB: a web-accessible database of experimentally determined protein-ligand binding affinities. Nucleic Acids Res 2007, 35:D198-D201.

31. Wang R, Fang X, Lu Y, Wang S: The PDBbind database: collection of binding affinities for protein-ligand complexes with known three-dimensional structures. J Med Chem 2004, 47:2977-2980.

32. Gao Z, Li H, Zhang H, Liu X, Kang L, Luo X, Zhu W, Chen K, Wang X, Jiang H: PDTD: a web-accessible protein database for drug target identification. BMC Bioinforma 2008, 9:104

33. Yuan Y, Pei J, Lai L: Binding site detection and druggability prediction of protein targets for structure- based drug design. Curr Pharm Des 2013, 19:2326-2333.

34. Li J, Zhang J, Chen J, Luo X, Zhu W, Shen J, Liu H, Shen X, Jiang H: Strategy for discovering chemical inhibitors of human cyclophilin A: focused library design, virtual screening, chemical synthesis and bioassay. J Comb Chem 2006, 8:326-337.

35. Wei D, Jiang X, Zhou L, Chen J, Chen Z, He C, Yang K, Liu Y, Pei J, Lai L: Discovery of multitarget inhibitors by combining molecular docking with common pharmacophore matching. J Med Chem 2008, 51:7882-7888.

36. Liu X, Xie H, Luo C, Tong L, Wang Y, Peng T, Ding J, Jiang H, Li H: Discovery and SAR of thiazolidine-2, 4-dione analogues as insulin-like growth factor-1 receptor (IGF-1R) inhibitors via hierarchical virtual screening. J Med Chem 2010, 53:2661-2665.

37. Chen Z, Wang X, Zhu W, Cao X, Tong L, Li H, Xie H, Xu Y, Tan S, Kuang D: Acenaphtho [1, 2-b] pyrrole-Based selective fibroblast growth factor receptors 1 (FGFR1) Inhibitors: design, synthesis, and biological activity. J Med Chem 2011, 54:3732-3745.

38. Liu X, Bai F, Ouyang S, Wang X, Li H, Jiang H: Cyndi: a multi-objective evolution algorithm based method for bioactive molecular conformational generation. BMC Bioinforma 2009, 10:101.

39. Bai F, Liu X, Li J, Zhang H, Jiang H, Wang X, Li H: Bioactive conformational generation of small molecules: a comparative analysis between force-field and multiple empirical criteria based methods. BMC Bioinforma 2010, 11:545.

40. Berman HM, Westbrook J, Feng Z, Gilliland G, Bhat TN, Weissig H, Shindyalov IN, Bourne PE: The protein data bank. Nucleic Acids Res 2000 28:235-242

41. Zhou W, Liu X, Tu Z, Zhang L, Ku X, Bai F, Zhao Z, Xu Y, Ding K, Li H: Discovery of Pteridin-7 (8 H)-one-based irreversible inhibitors targeting the epidermal growth factor receptor (EGFR) Kinase T790M/L858R mutant. J Med Chem 2013, 56:7821-7837.

42. Symyx Technologies. MDL drug data report: Sci Tegic Accelrys Inc., the MDL Drug Data Report (MDDR). In Database is available at http://www.accelrys.com/.

43. Dong G, Wang S, Miao Z, Yao J, Zhang Y, Guo Z, Zhang W, Sheng C: New tricks for an old natural product: discovery of highly potent evodiamine derivatives as novel antitumor agents by systemic structure-activity relationship analysis and biological evaluations. J Med Chem 2012, 55:7593-7613.

44. Rohrer SG, Baumann K: Maximum unbiased validation (MUV) data sets for virtual screening based on PubChem bioactivity data. J Chem Inf Model 2009, 49:169-184.
45. Mysinger MM, Carchia M, Irwin JJ, Shoichet BK: Directory of useful decoys, enhanced (DUD-E): better ligands and decoys for better benchmarking. J Med Chem 2012, 55:6582-6594.

46. Zou J, Xie H-Z, Yang S-Y, Chen J-J, Ren J-X, Wei Y-Q: Towards more accurate pharmacophore modeling: multicomplex-based comprehensive pharmacophore map and most-frequent-feature pharmacophore model of CDK2. J Mol Graph Model 2008, 27:430-438.

47. Sanders MP, Barbosa AJ, Zarzycka B, Nicolaes GA, Klomp JP, de Vlieg J, Del Rio A: Comparative analysis of pharmacophore screening tools. J Chem Inf Model 2012, 52:1607-1620.

48. de Medina P, Favre G, Poirot M: Multiple targeting by the antitumor drug tamoxifen: a structure-activity study. Curr Med Chem Anticancer Agents 2004, 4:491-508.

49. De Medina P, Paillasse MR, Segala G, Poirot M, Silvente-Poirot S: Identification and pharmacological characterization of cholesterol-5, 6-epoxide hydrolase as a target for tamoxifen and AEBS ligands. Proc Natl Acad Sci 2010, 107:13520-13525.

50. Ramaswamy B, Lu Y, Teng K-y, Nuovo G, Li X, Shapiro CL, Majumder S: Hedgehog signaling is a novel therapeutic target in tamoxifen-resistant breast cancer aberrantly activated by PI3K/AKT pathway. Cancer Res 2012, 72:5048-5059.

51. Paavonen T, Aronen H, PyrhÖNen S, Hajba A, Andersson L: The effect of toremifene therapy on serum immunoglobulin levels in breast cancer. Apmis 1991, 99:849-853.

52. Favoni RE, de Cupis A: Steroidal and nonsteroidal oestrogen antagonists in breast cancer: basic and clinical appraisal. Trends Pharmacol Sci 1998, 19:406-415

53. Santner SJ, Santen RJ: Inhibition of estrone sulfatase and 17ßhydroxysteroid dehydrogenase by antiestrogens. J Steroid Biochem Mol Biol 1993, 45:383-390.

54. Levine RM, Rubalcaba E, Lippman ME, Cowan KH: Effects of estrogen and tamoxifen on the regulation of dihydrofolate reductase gene expression in a human breast cancer cell line. Cancer Res 1985, 45:1644-1650.

55. Nuwaysir EF, Daggett DA, Jordan VC, Pitot HC: Phase II enzyme expression in rat liver in response to the antiestrogen tamoxifen. Cancer Res 1996, 56:3704-3710.

56. Ritchie GA: The direct inhibition of prostaglandin synthetase of human breast cancer tumor tissue by tamoxifen. Recent Results Cancer Res 1980, 71:96-101.

57. Fleming CD, Bencharit S, Edwards CC, Hyatt JL, Tsurkan L, Bai F, Fraga C, Morton CL, Howard-Williams EL, Potter PM: Structural insights into drug processing by human carboxylesterase 1: tamoxifen, mevastatin, and inhibition by benzil. J Mol Biol 2005, 352:165-177.

58. Rowlands MG, Budworth J, Jarman M, Hardcastle IR, McCague R, Gescher A: Comparison between inhibition of protein kinase $C$ and antagonism of calmodulin by tamoxifen analogues. Biochem Pharmacol 1995, 50:723-726.

59. Nilsson UW, Garvin S, Dabrosin C: MMP-2 and MMP-9 activity is regulated by estradiol and tamoxifen in cultured human breast cancer cells. Breast Cancer Res Ttreat 2007, 102:253-261.

60. Messiha FS: Leu-enkephalin, tamoxifen and ethanol interactions: effects on motility and hepatic ethanol metabolizing enzymes. Gen Pharmacol 1990, 21:45-48.

61. Lax E, Rumstadt F, Plasczyk H, Peetz A, Schriefers H: Antagonistic action of estrogens, flutamide, and human growth hormone on androgen-induced changes in the activities of some enzymes of hepatic steroid metabolism in the rat. Endocrinology 1983, 113:1043-1055.

doi:10.1186/1758-2946-6-28

Cite this article as: Wang et al: iDrug: a web-accessible and interactive drug discovery and design platform. Journal of Cheminformatics 2014 6:28. 